\title{
Der Zusammenhang zwischen den Variationsprinzipien der projektiven und der vierdimensionalen Relativitätstheorie
}

\author{
Von Günther Ludwig \\ Aus dem Institut für theoretische Physik der Universität Göttingen \\ (Z. Naturforschg. 2 a, 3-5 [1947]; eingegangen am 30. Aug. 1946)
}

\begin{abstract}
Es wird gezeigt, daß sich jedes Gleichungssystem, das man als Eu ler sche Gleichungen eines fünfdimensionalen Variationsprinzips der projektiven Relativitätstheorie erhält, sich ebenfalls aus einem entsprechenden vierdimensionalen Variationsprinzip herleiten läßt.
\end{abstract}

$\mathrm{N}$ achdem die projektive Relativitätstheorie durch die Arbeiten von W. Pa u li ${ }^{1}$ in ihrer Form einen gewissen Abschluß erreicht hatte, wurde neuerdings von P. J or da ${ }^{2}$ eine Erweiterung dieser Theorie gegeben, die darauf beruht, daß die projektive Invariante

$$
J=g_{\mu \nu} X^{\mu} X^{v}, \quad(\mu, \nu=0,1, \ldots 4)
$$

die bisher immer gleich 1 gesetzt wurde, als Funktion der Koordinaten $X^{\nu}$ angesehen wird. Es läßt sich dann $J$ bis auf konstante Faktoren als Gravitations-,,Konstante“ deuten, die dann keine Konstante mehr ist, sondern eine Feldfunktion. Dies ist von besonderer Bedeutung für die Entwicklung des Weltalls ${ }^{2}$.

Zur Aufstellung von Feldgleichungen geht man, wie es bisher in der allgemeinen Relativitätstheorie üblich war, von einem Variationsprinzip

$$
\delta \int \mathcal{L} d \tau=\delta \int L \sqrt{-g} d \tau=0
$$

aus, wobei $L$ eine projektive Invariante, $\mathbb{L}=$ $L \sqrt{-g}$ eine Invariantendichte ist. Wie üblich, ist

$$
g=\operatorname{Det}\left|g_{\mu \nu}\right| ; d \tau=d X^{0} d X^{1} \ldots d X^{4} .
$$

In $(2)$ ist $\sqrt{-g}$ geschrieben, da $g$ negativ ist.

Setzt man in (2) speziell $L=R$, dem projektiven Krümmungsskalar, so erhält man als Eulersche Gleichungen des Variationsprinzips (2) die Feldgleichungen des Vakuums ${ }^{2}$ :

$$
R_{\mu \nu}-1 / 2 g_{\mu \nu} R=0,
$$

1 W. Pauli, Ann. Physik V, 18, 305 ff. [1933].

${ }^{2}$ P. J or d a n u. Cl. M ü l l e r, Z. Naturforschg. 2 a, 1 [1947]. wobei $R_{u}$ der verjüngte Krümmungstensor ist. Durch Verjüngen von (4) folgt $R=0$ und damit aus (4):

$$
R_{1 ! v}=0 .
$$

Durch Übergang zur affinen Geometrie findet man aus (5) die Feldgleichungen für die Gravitationspotentiale $g_{i k}$, die elektromagnetischen $\mathrm{Po}$ tentiale $\varphi_{i}$ und die Gravitations-,,Konstante ${ }^{6} x^{3}$ :

$$
\begin{gathered}
\stackrel{4}{R}_{k}+\frac{\varkappa}{c^{2}} F_{j}^{l} F_{k l}=-\frac{1}{2 \varkappa}\left(\varkappa_{\|k\|_{j}}+\frac{\varkappa_{1 k} \varkappa_{1 j}}{2 \varkappa}\right) ; \\
F^{j k}=-\frac{3}{2} \frac{\varkappa_{1 j}}{\varkappa} F^{j k} ; \\
\frac{\varkappa}{c^{2}} F_{k l} F^{k l}+\frac{1}{2} \frac{\varkappa_{1 k} g^{k l} \varkappa_{1 l}}{\varkappa^{2}}-\frac{\varkappa_{\|k\| l} \mathrm{~g}^{k l}}{\varkappa}=0 .
\end{gathered}
$$

Hierbei sind $\stackrel{4}{R}$ und $\stackrel{4}{R}_{j k}$ der Krümmungsskalar bzw. verjüngte Krümmungstensor der vierdimensionalen affinen Geometrie. $F_{k l}$ sind die elektromagnetischen Feldstärken:

$$
F_{k l}=\varphi_{l \mid l}-\varphi_{k \mid l} .
$$

Es ist nun bemerkenswert, daß man jedes Variationsprinzip (2) in ein vierdimensionales affines überführen kann, so daß man z. B. die Feldgleichungen (6) bis (8) auch direkt aus einem vierdimensionalen Variationsprinzip erhalten kann.

${ }^{3}$ Dabei bedeuten $\mathfrak{f}_{\mid k}=\partial \mathfrak{f} / \partial x^{k}$ die gewöhnlicheDifferentiation und entsprechend z. B. $\mathfrak{F}^{j k}|| l$ die kovariante Differentiation nach $x^{l}$. 
Um dies zu zeigen', formen wir ganz allgemein ein fünfdimensionales Integral .

$$
W=\int L \sqrt{-g} d \tau
$$

in ein vierdimensionales $\mathrm{um}$. Zu diesem Zweck führen wir in (10) als neue Integrationsvariable die affinen Koordinaten

$$
x^{k}=x^{k}\left(X^{0}, \ldots X^{t}\right) \quad(k=1,2, \ldots 4)
$$

und eine beliebige Funktion

$$
\eta=\eta\left(X^{0}, \ldots X^{\mathbf{4}}\right)
$$

homogen vom ersten Grade in den $X^{0} \ldots X^{4}$ ein. Wir wollen die übliche Bezeichnungsweise ${ }^{4}$ einführen:

$$
g_{v}^{k}=x_{\mid \nu}^{k} ; g_{k}^{\nu}=g_{k i} g_{\mu}^{i} g^{\mu \nu}
$$

Da die $x^{k}$ homogene Funktionen 0-ten Grades sind, gilt

$$
g_{v}^{k} X^{v}=0
$$

Aus (14) und (13) folgt dann auch

Fuir $\eta$ gilt:

$$
g_{k}^{v} X_{v}=0
$$

$$
\eta_{, \mu} X^{\prime \prime}=\eta
$$

Die Funktionaldeterminante

$$
\frac{\partial\left(\eta, x^{1} \ldots x^{4}\right)}{\partial\left(X^{0}, \ldots X^{4}\right)}=\operatorname{Det}\left|\begin{array}{c}
\left(\eta_{1 \mu}\right) \\
\left(g_{\mu}^{k}\right)
\end{array}\right|
$$

läßst sich unter Berücksichtigung von (14) und (16) z. B. durch Addition geeigneter Vielfachen der vier letzten Spalten zur ersten umformen zu:

$$
\frac{\partial\left(\eta, x^{1}, \ldots x^{4}\right)}{\partial\left(X^{0}, \ldots X^{4}\right)}=\eta \frac{\Delta^{0}}{X^{0}}
$$

wobei $A^{0}$ die Minore in (17) $\mathrm{zu} \eta_{10}$ ist. Setzt man für die affinen Koordinaten

so wird

$$
d x^{1} \ldots d x^{4}=d \stackrel{4}{\tau}
$$

$$
W=\int L \sqrt{-g} \frac{X^{\nu}}{\Delta^{0}} d \log \eta d \tau .
$$

Wir betrachten nun die Determinante

$$
D=\operatorname{Det}\left|\begin{array}{c}
\left(X_{\mu}\right) \\
\left(g_{\mu}^{k}\right)
\end{array}\right|
$$

Genau wie bei (17) berechnet man unter Berücksichtigung von (1) und (14) leicht:

$$
D=J \frac{\Delta^{0}}{X^{0}}
$$

Setzt man zur Abkürzung $g_{\mu}^{k}=g_{\nu}^{k} g^{\nu \mu}$, so ist

$D^{\prime}=\operatorname{Det}\left|\begin{array}{c}\left(X^{\mu}\right) \\ \left(g^{k} \mu\right)\end{array}\right|=\operatorname{Det}\left|\begin{array}{c}\left(X_{\mu}\right) \\ \left(g_{\mu}^{k}\right)\end{array}\right| \operatorname{Det}\left|g^{\mu \nu} \cdot\right|=\frac{D}{g}$.

Aus (21) und (23) folgt sofort:

$$
D D^{\prime}=J \cdot \operatorname{Det}\left|g^{i k}\right|=\frac{J}{g}=\frac{D^{2}}{g},
$$

wenn man mit $\stackrel{4}{g}$ die Determinante aus dem affinen metrischen Tensor bezeichnet:

$$
\stackrel{4}{g}=\left|g_{i k}\right| \text {. }
$$

Aus (24) und (22) folgt schließlich ${ }^{5}$ :

$$
\sqrt{-g}=J^{1 / 2} \frac{\Delta^{0}}{X^{0}} \sqrt{-g^{4}} .
$$

(26) in (20) eingesetzt ergibt:

$$
W=\int L J^{1 / 2} \sqrt{-\frac{4}{g}} d \tau d \log \eta .
$$

Da der Integrand $L J^{1 / 2}$ eine Invariante ist, ist er homogen vom 0 -ten Grade. Er hängt daher nur von den $x^{k}$ und nicht von $\eta$ ab, so daß über $\eta$ ausintegriert werden kann. Ist $\eta^{\prime}$ der Wert von $\eta$ an der oberen, $\eta^{\prime \prime}$ der an der unteren Grenze des fünfdimensionalen Bereiches, über den (27) zu integrieren ist, so ist $\eta^{\prime} / \eta^{\prime \prime}$ homogen vom 0 -ten Grade und damit nur Funktion der $x^{k}$. Es ist

$$
\frac{\eta^{\prime}}{\eta^{\prime \prime}}=\frac{X^{0 \prime}}{X^{0 \prime \prime}}=\ldots=\frac{X^{4 \prime}}{X^{4 \prime \prime}}=\lambda\left(x^{k}\right)
$$

${ }^{4}$ P. Jordan, Physik. Z. 45 [1944] (Korrekturfahnen).

${ }^{5} \mathrm{Da}$ die erste Spalte in (17) oder (21) vor den anderen nicht ausgezeichnet ist, ergibt sich:

$$
\frac{\Delta^{0}}{X^{0}}=\frac{\Delta^{1}}{X^{1}}=\ldots=\frac{\Delta^{4}}{X^{4}}=a
$$

und hieraus $\Delta^{v}=a X^{\nu}$ und $\Delta^{v} \dot{X}_{\nu}=a J$, d. h.

$$
a=J^{-1} \Delta^{v} X_{v} \text {. }
$$


das Verhältnis der Koordinaten der oberen zur unteren Grenze. Aus (27) erhält man dann das vierdimensionale Integral

$$
W=\int L J^{1 / 2} \log \lambda \sqrt{-g^{4}} d \tau
$$

$\mathrm{Zu}$ jedem Weltpunkt $x^{k}$ gehört ein Strahl $\varrho X^{\mu}$ im fünfdimensionalen $X^{\nu}$ Raum. Das Gebiet, über das in (27) $\mathrm{zu}$ integrieren ist, schneidet aus jedem Strahl ein Stück aus, und $\log \lambda\left(x^{k}\right)$ ist ein Maß für die Länge des ausgeschnittenen Stückes, auch dann, wenn dieses nicht zusammenhängend ist.

$\mathrm{Da}$ in $\lambda$ nicht die Feldvariablen eingehen, sind die beiden Variationsprobleme

$$
\delta \int L \sqrt{-g} d \tau=0 ; \delta \int L J^{1 / 2} \sqrt{-\stackrel{4}{g}} d \tau=0
$$

äquivalent. Ist insbesondere $L=R$, so ergibt sich, daß die Feldgleichungen (6) bis (8) auch eine Folge des Variationsprinzips

$$
\delta \int R J^{1 / 2} \cdot \sqrt{-\stackrel{4}{g}} d \tau=0
$$

sind. Nun läßt $\operatorname{sich} R \operatorname{durch} \stackrel{4}{R}, F_{k l}$ und $x$ ausdrücken ${ }^{4}$ :

$$
\begin{aligned}
R=R^{R} & +\frac{1}{2} \frac{\varkappa}{c^{2}} F_{k l} F^{k l}-\frac{\varkappa_{\mid k} \mathrm{~g}^{k l} \varkappa_{1 l}{ }^{?}}{2 \varkappa^{2}} \\
& +\frac{\varkappa_{\|k\| l} g^{l k}}{\varkappa}
\end{aligned}
$$

so daß (31) übergeht in:

$$
\begin{aligned}
\delta \int \sqrt{x}(\stackrel{4}{R} & \left.+\frac{1}{2} \frac{x^{2}}{c^{2}} F_{k l} F^{k l}-\frac{\varkappa_{14} g^{k l} x_{1 l}}{2 x^{2}} \cdot \frac{x_{H k \| l} g^{l k}}{\varkappa}\right) \sqrt{-g} \cdot d \tau=0 . \\
& +\frac{4}{4}=0 .
\end{aligned}
$$

Als einfacheres weiteres Beispiel betrachten wir das Variationsprinzip

$$
\delta \int\left(b^{\mu} \varphi_{\mid \mu}+a \varphi\right) \sqrt{-g^{4}} d \dot{4}=0,
$$

wobei $\varphi$ zu variieren ist. Man erhält als E u l e r sche Gleichungen:

$$
a=b^{\mu}{ }_{\| \mu} \text {. }
$$

Andererseits ist mit (34) das vierdimensionale Variationsprinzip:

$$
\delta \int J^{1 / 2}\left(b^{k} \varphi_{1 k}+a \varphi\right) \sqrt{-\stackrel{4}{g}} d \tau=0 .
$$

äquivalent, woraus man erhält:

$$
a=J^{1 / 2}\left(J^{1 / 2} b^{k}\right)_{\| k} .
$$

Durch Vergleich mit (35) folgt dann sofort:

$$
b^{\mu ! u}=b_{\| k}^{k}+\frac{1}{2} \frac{J_{1 k} b^{k}}{J},
$$

womit die fünfdimensionale Divergenz durch die vierdimensionale ausgedrückt ist. Setzt man speziell $b^{\mu}=\psi_{\mid v} g^{\nu \mu}$, , so folgt:

$$
\begin{aligned}
\Delta \Psi=\Psi_{\|\mu\| \nu} g^{v \mu} & =\Psi_{\Downarrow k k_{1, i}} g_{-}^{i k}+\frac{1}{2} \frac{J_{1 k}}{J} \Psi_{1 i} g^{i k} \\
& =\Delta \psi+\frac{1}{2} \frac{J_{\mid k}}{J} \Psi_{\mid i} g^{i k},
\end{aligned}
$$

wobei $\Delta$ der fünfdimensionale und $\Delta^{4}$ der vierdimensionale L a p l a c e sche Operator ist. Damit haben wir eine neue, sehr vereinfachte Herleitung der Gl. $(5,21)$ aus Jord a n ${ }^{4}$.

Die Arbeit entstand im Zusammenhang einer Untersuchung allgemeiner Feldgleichungen bei veränderlicher Gravitationskonstante, die vor dem Abschluß steht und für deren Anregung und Förderung ich Hrn. Prof. Dr. Jordan herzlich danke. 